\title{
Wholeness as a Conceptual Foundation of Physical Theories
}

\author{
Barbara Piechocinska \\ Uppsala University, Box 534, 75121 Uppsala, Sweden
}

(Dated: July 15, 2018)

\begin{abstract}
A description of physical reality in which wholeness is the foundation is discussed along with the motivation for such an attempt. As a possible mathematical framework within which a physical theory based on wholeness may be expressed, elementary embeddings along with the Wholeness Axiom are suggested. It is shown how features of wholeness such as wholeness being indescribable, more than the sum of parts, locally accessible and giving rise to a self-similar, or holographic, type of order are reflected in the mathematics. It is also shown how all the sets in the mathematical universe may be expressed as emerging from the dynamics of wholeness. Moreover, it is indicated how the mathematics may be further developed so as to connect up with a physical interpretation.

Keywords: wholeness, Wholeness Axiom, Laver sequence, implicate order, braids, left-distributivity
\end{abstract}

\section{BACKGROUND AND MOTIVATION}

The purely reductionist approach to physics, in which the world is modelled as being made up of separate identifiable parts that interact, has proven successful over the centuries. It was not until the beginning of the twentieth century that experimental data demanded a drastically new theory. To accommodate the new results the quantum theory was invented. Despite the deeply rooted reductionist tradition scientists discovered early on that the quantum theory was not purely reductionistic, and yet it was approached and formed from the classical side. It is for instance normal procedure to attempt to quantize classical systems, while it could be argued that the quantum theory, being more fundamental than classical theories, should be the starting point.

The new aspects, not reducible to a purely reductionist perspective, were intimately related to what Bohr called wholeness. From the invention of the quantum theory Bohr has been clear to point out the key role that wholeness plays [1]. When two quantum systems interact, or have interacted, we can in general no longer view the system of interaction as being made up of two separately existent parts. Instead an inseparable totality is formed. The wave function of such an interacting system can, in general, not be expressed as a product of the wave functions of the two previously separated systems. And so a reductionist picture based on the interactions of static identifiable parts with definite properties cannot be maintained. The details of how a nonlocal, indivisible, and dynamical form of wholeness plays a central role are presented in the Ontological Interpretation of Quantum Theory [2] and a short summary of them will be given in the Section 2. The effects of a correlation related to the indivisible wholeness of quantum systems that transcends space and time (entanglement) have been objectively demonstrated through experiments [3]. Failing to conform to reductionism and being essential in the foundations of the quantum theory, it would be both intellectually satisfactory and of instrumental value in future research to have an understanding of what philosophical ideas wholeness entails.
Since the advent of the quantum theory severe problems have arisen when attempting to form a consistent, unified description through physics. There appear to be incompatibilities between fundamental physical theories such as the quantum theory and the theory of general relativity. In attempting to find a common ground for both of them various physicists have suggested wholeness [4] (chapters 5-6), 2] (chapter 15), 5]. The prospect of being a common factor in both the quantum theory and the theory of general relativity hints at the possibility of leading to a theory that transcends and includes the both of them.

In view of the present incompatibilities, the difficulty to fit contemporary physics into a reductionist framework, and the indications that a non-reductionistic wholeness is essential in physics, it is suggestive that we attempt a scientific description that starts from wholeness. However, in order to engage in such an attempt we are in considerable need of new ways of thinking (fundamental concepts) as well as new mathematical tools, all due to the particularly elusive nature of wholeness. This paper addresses that need and presents a proposition for new foundations of physics with the fundamental concepts and a mathematical theory capable of representing them. In particular it proposes a concrete mathematical description of Bohm's implicate order ${ }^{1}$ that is based on a new axiom in Set Theory. It further proposes that the connection from the mathematically described implicate order to physics be made through the left-distributive algebra that this axiom generates, and in particular through braids.

The paper is set up in the following way. We shall start, in Section 2, by summarizing the work of Bohm and Hiley in order to give a specific example of how wholeness comes into the quantum theory. Then we shall continue, in Section 3, by taking a closer look at the concept of wholeness in general and at the particular feature of wholeness that will serve as the fundamental concept

\footnotetext{
${ }^{1}$ see Section 2.B for implicate order
} 
which will be represented mathematically. We will then proceed to Section 4 in which an example of a mathematical framework capable of expressing the fundamental concept and key features of wholeness will be given. After that we shall, in Section 5, look at some examples of how we expect the approach based on starting from wholeness to be useful. In particular, we shall see how wholeness facilitates the understanding of the properties of the quantum theory, and indicate what mathematical structures (used in physics) naturally arise from the proposed mathematics. Finally, in Section 6, we shall sum up the conclusions.

\section{EXAMPLE OF WHOLENESS IN THE QUANTUM THEORY}

When attempting to study physics in search of the underlying concepts we find ourselves to be quite fortunate because there are scientists who have already done a substantial amount of work in this direction. Of particular interest is the work of Bohm, Hiley, and collaborators. A large part of their work originates in intuitions deduced from Bohm's interpretation of the quantum theory [6], [2], 7]. One of the great advantages of this interpretation is that it allows for an imaginative and intuitive understanding of quantum phenomena and thereby opens up the door to further insights that may become crucial for an extension of the quantum theory, possibly moving into a new theoretical framework. In particular, through the quantum potential, which as we shall see can be derived from the Schrödinger equation, this interpretation explicitly accentuates the essential role of a dynamical, unbroken wholeness. We shall now go into slightly greater detail in order to better see the holistic aspect of physics and how they are suggestive of Bohm's implicate order. The starting point will be Bohm's interpretation of the quantum theory.

\section{A. Bohm's interpretation of the quantum theory}

From being a statistical theory about the outcomes of measurements in which the actual phenomena involved cannot be analyzed [8] (p. 72), Bohm developed an essentially ontological interpretation of the quantum theory that provides intuitive understanding and further insight into the actuality of systems. In particular, by using the Hamilton-Jacobi theory, it provides a framework within which classical and quantum effects can be contrasted. Such comparisons offer a richer understanding of what Niels Bohr called the unanalyzable wholeness [1] and lead to the development of Bohm's implicate order
2. It should also be mentioned that aside from providing an ontology for, and an intuitive understanding of the quantum theory, Bohm's interpretation also gives an account for each individual quantum process and accounts for measurements.

In Bohm's view, the wave function is not regarded as a complete representation of a quantum state. Its interpretation is not only probabilistic, such as the square root of the probability of finding a particle in a particular region. Instead the wave function is an actual quantum field ( $\psi$-field) that provides a partial description of the formative cause, as well as a function from which actual probabilities can be derived [10]. This treatment of the wave function is what eventually leads to an account of measurement.

In the original interpretation of Bohm [6], a particle is assumed to exist with a well defined position and momentum. One can therefore say that the particle in fact moves along a definite trajectory. The particle has a quantum field ( $\psi$-field) associated with it. They are physically inseparable. On a deeper level of the ontology the quantum field and the particle are considered by Bohm to be different aspects of the same process [2] (sec.15.8).

Now Bohm resorts to Hamilton-Jacobi theory, which can be seen as a theory of the interrelationships of rays and waves [7] (chapter 2). This treatment produces a picture in which one can see how both classical and quantum effects arise. In order to see this in the non-relativistic case, one starts with the wave function written in its polar form

$$
\psi=R(\mathbf{x}, t) e^{i S(\mathbf{x}, t) / \hbar},
$$

where both $S$, the phase of $\psi$, and $R$, its amplitude, are real fields that are dependent on the position, $\mathbf{x}$, and the time parameter $t$, and where $\hbar=h /(2 \pi)$, $h$ being Planck's constant. The next step is to derive the Quantum Hamilton-Jacobi equation from the Schrödinger equation. Doing this for one quantum particle one goes through the following steps. We start with the Schrödinger equation, which describes the time evolution of the quantum system,

$$
i \hbar \frac{\partial \psi}{\partial t}=-\frac{\hbar^{2}}{2 m} \nabla^{2} \psi+U \psi,
$$

where $m$ is the mass of the particle and $U=U(\mathbf{x}, t)$ is a (real) classical potential. We now substitute (11) into (2), work out the Laplacian, rearrange the terms and divide them up into two equations, one for the real part and the other for the imaginary part. The real part then yields

$$
\frac{\partial S}{\partial t}+\frac{(\nabla S)^{2}}{2 m}+U+Q=0
$$

\footnotetext{
2 Consequently, the implicate order can be seen as providing a kind of understanding of the wholeness found in the quantum theory that has the capability of going beyond the quantum theory [9].
} 
where $U$ is still a classical potential and $Q$ is the quantum potential. More explicitly, we see that

$$
Q=-\frac{\hbar^{2}}{2 m} \frac{\nabla^{2} R}{R} .
$$

When $Q \rightarrow 0$ we move from the quantum domain towards the classical limit [7](p. 225) and for $Q=0$ equation (3) becomes the classical Hamilton-Jacobi equation. If we see the quantum potential as a type of potential energy we can view (31) as an extended version of the conservation of energy, valid in the quantum domain. In order to calculate the momentum of the quantum particle we use the guidance condition ${ }^{3}$

$$
p=\nabla S
$$

The imaginary part of (11) in (2) is

$$
\frac{\partial R^{2}}{\partial t}=-\nabla\left(R^{2} \frac{\nabla S}{m}\right)
$$

This can be seen as an equation for the conservation of probability.

The above approach grants us the unique opportunity of studying the quantum potential, which is responsible for the non-classical effects of the quantum theory. If we can understand its nature and physical relevance, we will come one step closer to intuitively understanding quantum phenomena and possibly going beyond them.

In order to pursue this we shall now stress how radically different the quantum potential is from classical potentials. Its dynamics goes beyond what can be seen as a mechanical interaction of external parts. To start with, we note that the quantum potential has no external source from which it can be seen to emanate, such as for instance the gravitational potential could be seen to emanate from a massive body in space. This is basically due to the fact that the quantum potential is constructed from the $\psi$-field, which itself depends on the whole system and lacks an external source. And so, the quantum potential's dependence on the $\psi$-field introduces an irreducible dependence on the entire environment. When more particles are involved it becomes even more clear that the wave function depends on the whole system. Here we encounter a holistic aspect in which wholeness is more than just all the parts and their interactions.

Moreover we note from equation (4) that the quantum potential has a different form than classical potentials. We find $R$ both in the numerator and denominator,

\footnotetext{
${ }^{3}$ It should be mentioned that an approach based on this condition was introduced by other authors as "Bohmian Mechanics" [1] [12]. The mathematics of it is in accordance with Bohm's own approach. However, several parts, originally existing in Bohm's interpretation and crucial to its philosophy (such as the quantum potential), have been omitted. For a short comparison between the two approaches, see [13].
}

which means that the quantum potential does not in general necessarily diminish with distance. Even though the $\psi$-field may go to zero as the distance increases, the quantum potential does not have to diminish. This means that remote features may have significant influence on particle movement. When more particles are involved, this can give rise to the phenomenon of non-locality. The quantum potential will in general depend on the positions of all $N$ particles,

$$
Q=-\frac{\hbar^{2}}{2 m} \frac{\nabla^{2} R\left(x_{1}, x_{2}, \ldots, x_{N}\right)}{R\left(x_{1}, x_{2}, \ldots, x_{N}\right)}
$$

so that the quantum force $-\nabla_{k} Q$ on some particle $k$ depends on all particles.

Furthermore we note that it is not the intensity of the $\psi$-field that regulates the effect it has on the movement of a particle but its form. This again is due to the presence of $R$ in the denominator. An increase in the amplitude, by some factor $c$, has no bearing on the quantum potential, as $c$ divides out. This in turn suggests that the action of $\psi$ on the particle, through $Q$, is of a different kind than classical pushing or pulling (a mechanical transfer of momentum and energy), and is thoroughly discussed by Bohm and Hiley [2]. They suggest that the quantum field be treated as an information potential. The usage of the word information is different [13] from Shannon's information 14], which refers to our ignorance or certainty about a system. Instead, Bohm and Hiley's information "in-forms," actively puts form into. The information in the quantum potential guides a particle along its way. It is information relevant to the objective movement of a particle, information that has meaning to the particle. When it guides a particle it is referred to as "active information." Bohm and Hiley have suggested the metaphor of a boat being guided by the radar. The actual waves of the radar do not push or pull the boat, as do the waves of the sea. In a similar way the quantum potential guides through information that is meaningful to the particle, by putting form into the motion and not by pushing it.

\section{B. Dynamical unfoldment and enfoldment and the implicate order}

The dynamics suggested by the quantum potential clearly indicates a radical change from the classical framework. The quantum potential appears to suggest a dynamics where the irreducible totality of a system and its environment acts (from beyond any particular spatial source) and forms the explicit movement of some particle. In other words, the lack of an external local source for the quantum potential along with its irreducible dependence on the whole system and, in general the existence of non-locality, suggests that it operates from an irreducible order beyond space and time. Bohm called this the implicate order. The implicate order is dominated by its holistic aspects and can be seen as a dynamical totality where things cannot be distinguished from each other 
and are instead inseparably intertwined. In other words, despite a lack of crystallization, or definite boarders and distinguishability (that we are used to seeing in space), all the relationships are intact and preserved.

Operating from the implicate order the quantum potential gives explicit form to the movement of a particle. Here we can talk about the explicate order. The explicate order is the order where things can be distinguished from each other, or seen to exist outside of each other, and approximated as independent.

An important fact here is that in order to form the movement of the particle the $\psi$-field needs information from the whole environment that is processed as a totality. We see how the implicate order, which is beyond the visible (explicate), plays an essential, formative role in the dynamics. And so, the movement of the part comes out of the totality, not the other way around. Since the totality is irreducible we cannot reduce it to parts. This is how wholeness is greater than the sum of its parts and needs to be considered in any serious attempt to go beyond the quantum theory. This is the fundamental idea upon which we will continue to build and why we shall try to derive the movement of parts from the dynamics of wholeness instead of starting with parts and letting their sum describe a reductionistic wholeness.

To do this we shall need to discuss the nature of the process of going from the implicate order to the explicate order and the other way around. This will be done in terms of unfoldment and enfoldment. The dynamical concepts of unfoldment and enfoldment were introduced and developed by Bohm [4] in an effort to provide a common basis capable of accounting for both quantum and relativistic effects. The implicate order is the enfolded order while the explicate order is the unfolded order. To explain these aspects Bohm proposes an illustrative metaphor. One places a cylinder container with a smaller radius inside of a fixed one with a larger radius. Then one pours glycerine between them. On top of the glycerine one then places a drop of ink. If one at this point starts turning the inner cylinder the ink-drop will smear out and become a line. After a while no ink will be visible. This is analogous to the enfoldment process. The ink-drop was first explicit and has now become enfolded into the order of the molecules and is implicit. Though the drop cannot be seen explicitly it is still there, implicitly in the order. If we now start turning the inner cylinder in the other direction the ink-drop will appear again. This is then analogous to the unfoldment process. The ink-drop becomes explicit again. This example descriptively expounds the ideas of unfoldment and enfoldment but should not be taken literally.

The idea that Bohm tried to convey was that every thing is in some sense enfolded into the whole and that the whole is unfolded into every thing. Bohm called this dynamics the holomovement which is a holistic pulsation in which orders unfold and enfold. Emphasis should be put on the fact that this fundamental process is not a movement within space-time (like in the example of the ink-drop) but rather a process in which ultimately spacetime and its contents are created ${ }^{4}$. Recall that the quantum potential does not operate from any particular part in space but originates in an irreducible totality, and yet is locally accessible from any point within space where it puts explicit form into the movement. Likewise, the fundamental process originates in the implicate order which unfolds into an explicate order and forms it through the unfoldment. Because of the wording one might be inclined to think that there exists an implicate order and an explicate order and that they interact through unfoldment and enfoldment. This, however, is not what is being proposed here. Instead, focus should be put on the process, or movement. It is the nature of the unfoldment and enfoldment that is such that one can see it as giving rise to the explicate from the implicate. The explicate order does not have a permanent and independent existence. It is continually being created and dissolved. And the existence of the implicate order is of a subtle kind, it cannot be said to exist explicitly because it is implicit and beyond space and time. Therefore, it is suggested, movement is fundamental and can be expressed in terms of the enfoldment and unfoldment.

It has further been argued by Bohm and Hiley, in [2] (chapter 15.3), that essentially all the quantum mechanical laws of movement can already be seen as unfoldment and enfoldment. The value of a wave function at a particular time and point in space depends on the whole space at previous moments. Consequently a particular region can be said to enfold contributions from the whole space. Then, as it evolves, it unfolds into the whole space. The authors also show how Huygen's principle and Feynman diagrams can be understood in terms of enfoldment and unfoldment. Huygen's principle tells us that the propagation of a wavefront can be seen as each point on the wavefront acting as a wave-emitting source point. In other words, each point enfolds contributions from all other points on the wavefront and then unfolds, giving rise to a new wavefront. Since Feynman diagrams can be considered as based on Huygen's principle 2](p. 355), enfoldment and unfoldment can be considered as fundamental for them as well.

Summarizing Section 2 we may say that the essential concept that can be extracted from Bohm and Hiley's work is that there is a dynamical, unbroken wholeness. The dynamics seems to consist of unfoldments and enfoldments of explicate and implicate orders.

\footnotetext{
4 To see how the contents are created one extends Bohm's interpretation to the quantum field theory where a super-quantum potential affects the field equations in a non-local and non-linear way. There, it is not only the motion of a particle that is formed but the very existence of the particle itself.
} 


\section{DESCRIPTION FROM WHOLENESS}

\section{A. The concept of wholeness}

Starting with wholeness presents a particular challenge for a physical description of reality. This is due to its indefinable and elusive nature. Therefore, the approach taken here will be to assume the existence of an indefinable wholeness and investigate what conclusions this assumption may lead to in terms of physical theories. However, before making such an assumption some features of wholeness will be highlighted.

Wholeness is taken to be the ontological basis for the physically measurable reality. It is viewed as the totality that implicitly unites and gives rise to everything observable, but is more than the observable. In particular we will be dealing with a wholeness that is larger than the sum of its parts. Working with parts towards wholeness not only leaves us with an insufficient [15] and severely limited picture of wholeness, but also with one that is inconsistent $^{5}$. Furthermore, we do not wish to confine ourselves to working with parts because we are interested in describing a wholeness that is similar to, or at least not more limited than, the wholeness found in the quantum theory (see Sections 1 and 2).

Another central aspect of wholeness is that it is indefinable. This can be intuitively understood in the following way. Being whole is not in opposition to anything, not even to part. If wholeness were opposed to part and thereby excluded part it would not be whole. And so, it is not possible to gain a true understanding of wholeness based on division, or differentiation, and opposition. Consequently a description of wholeness is not possible in terms of our language because it is based on opposition and differentiation [17], 4] (chapter 3), 18]. Differentiation and opposition lead to definitions and to information that is based on the concept of lack of knowledge. To define something is to delimit it and say that it is that which is found within the limit and not that which is found outside of the limit. Such a delimitation cannot be performed on wholeness and one can therefore say that wholeness is not definable. In fact, trying to describe wholeness may be likened to attempting to reach infinity by, say, counting all the natural numbers, $1,2,3, \ldots$. No matter what number we reach it will not be infinity. Just like infinity is not a number, wholeness is not a part, or reducible to a description. As a consequence we see that any description is a limitation. This must also hold for a strictly holistic view of wholeness where it is seen as undivided and forever non-separable into individual modes. In what follows we shall therefore take the broader view that the holistic perspective and the reductionistic perspective are complementary in the

\footnotetext{
${ }^{5}$ Publications regarding the whole being more and different from the parts can be found in [16], 15].
}

sense that both are required in a description of reality based on the assumption that there is wholeness.

The relationship between the holistic and reductionistic aspects can be described as according to a self-similar, or holographic type of order (the order of Bohm's implicate order as described in the previous section). To see the self-similarity and holography we must first recognize that the ontological basis of each part is the holistic wholeness. In other words, one part implicitly contains all parts at its ground and all parts implicitly contain that one part, at their ground. Wholeness is in each explicit part, as that parts innermost nature or ontological ground, while from the implicate (holistic) perspective each part is inseparable from wholeness. And so, wholeness is (in) each part and each part is (in) wholeness. This can be seen as self-similar because through wholeness there is a similarity between a part and all parts, and holographic, because each part implicitly contains information from all parts. This kind of order is a consequence of the holistic properties and is already found in the quantum theory, where we see how the dynamics of a quantum system has an irreducible (holistic) dependence on its surroundings 2].

One should bear in mind that wholeness is not some physically measurable quantity and its existence can therefore not be directly, physically, verified through objective experiments. Instead it is the source of the physically measurable quantities. An analogy can be made to superposition in the quantum theory, which can never be explicitly measured. As described above, wholeness is qualitatively different from part or thing for instance in that it cannot be explicitly defined. However, we shall see that if wholeness is assumed a consistent picture of reality will take form in which wholeness, through its nature, may be seen as the source of physical reality. Perhaps just like the acceptance of infinities in mathematics provides a rigorous foundation for and definition of real numbers, an assumption of wholeness might account for the existence of the physical universe.

In order to provide a rigorous mathematical description for a physical theory that assumes the existence of wholeness we will need to find and specify what we consider to be fundamental concepts. Then, we will need to find a way of mathematically expressing them. Since the existence of wholeness is assumed, it is vital that this be reflected in the mathematical description on a fundamental level. We will now quickly review our understanding of a dynamical wholeness in order to provide the fundamental concept.

\section{B. The fundamental concept}

The fundamental concept, upon which we shall base our description of the physical universe is movement, or as suggested by Bohm [4], process. Besides the motivation provided by Bohm's approach (in Section 2) this can be motivated in the following way. In our descrip- 
tion of the physical universe we wish to take wholeness as fundamental. This requires our description to be complementary. We need both the holistic perspective and the reductionistic perspective. Although a description in terms of parts is certainly possible in the reductionist spirit, it cannot be a fundamental base for a description from wholeness that includes the holistic perspective.

Here is a slightly different way of seeing why parts may not be the best way of describing physical reality from wholeness. For a long time physicists have been trying to find a fundamental building block, entity, or part. Let us assume that such a thing exists and see what properties it should possess. However, let us not limit ourselves to building blocks that have positions or extensions in space or time. Suppose we find two such fundamental building blocks, then we will find that, the blocks being truly fundamental, we cannot make any kind of distinction between them. If no such distinction exists (not even a distinction in time or space) we conclude that the two fundamental building blocks are one. Consequently, we can say that there is only one fundamental building block for everything. This can be interpreted in two ways. The fundamental building block is either nothing or it is wholeness. Neither of these cases is consistent with a reductionist framework where identifiable parts are taken as fundamental. One reason is that neither nothing nor wholeness have outer limits which would allow us to define or identify them. Instead they are thing-less. And so, they may not be considered as parts.

As parts do not meet our expectations in terms of fundamental concepts for a description from wholeness we turn to movement or process as a plausible alternative. This movement or process should in its totality not be viewed as confined to physical space and time and is not reducible to a description of some parts that move in a sequential manner. Instead it is the fundamental dynamics of wholeness, and that which through self-limitation gives rise to physical space and time along with parts which may be viewed as properties of, or invariances in, the movement. And so, the dynamics is such that any part, and the change of any part, may be traced back to the fundamental movement. Such a dynamics needs to be wholeness preserving. Here follows an explanation. Wholeness may be said to be a totality implicitly containing everything. This in turn appears to imply that for it to be truly whole it cannot change. If it were to change and become different, then this would imply that it was not truly whole in the first place because it did not contain itself after the change. At the same time we know that change, at least in our physical world, exists. Therefore, we are looking for a dynamical description that allows wholeness to remain essentially unchanged though moving, from the perspective of parts, and thereby allowing for change. We plan to capture this dynamical feature of wholeness, the wholeness preserving movement, mathematically in a fundamental way. This will be the fundamental concept. And so essentially, the proposition here is to describe our physical reality as an expression of the dynamics of wholeness.

Furthermore, we should like to have a way of expressing diversification, preferably as a result of the wholeness preserving movement. We also expect to have the possibility of expressing time, space, physical objects as properties of or invariances in the movement.

\section{MATHEMATICAL FRAMEWORK}

In order to provide a mathematical foundation that is capable of expressing features of wholeness at the very fundamental level, it is suggested that we start with set theory, since most of mathematics can be expressed in terms of it, and add the Wholeness Axiom introduced by Corazza [19] to the normal axioms of set theory. The normal axioms of set theory are the Zermelo-Fraenkel set of axioms and the Axiom of Choice, abbreviated ZFC [20], [21], 22]. For approximatively eighty years it has been known that most of mathematics is derivable from them. These axioms refer to sets and are formulated in the formal language $\{\in\}$. This means that the only relation used in the formulation of the axioms is the membership relation, $\in$.

The mathematical theory within which we shall attempt to describe a theory from wholeness will contain the ZFC set of axioms together with the Wholeness Axiom and will be referred to as ZFC+WA. We will now proceed to see what the Wholeness Axiom is and what features of wholeness ZFC+WA is capable of expressing.

\section{A. The mathematical universe $V$}

An important concept for our purposes is the mathematical universe. The mathematical universe, $V$, is a proper class and can be expressed as:

$$
V=V_{0} \bigcup V_{1} \bigcup V_{2} \bigcup \ldots
$$

where the different stages are $V_{0}=\emptyset, V_{1}=P\left(V_{0}\right), V_{2}=$ $P\left(V_{1}\right) \ldots$, with $P()$ denoting the power set (the set of all subsets). It is interesting to note that there is a salient analogy between the mathematical universe $V$ and wholeness. As previously mentioned, wholeness implicitly contains everything but is more than the sum of the parts, and is itself not an explicitly existent entity (a part). The nature of its existence is implicit. In a similar way $V$ may be said to contain all the mathematically existent parts and yet be more than their sum and not itself an explicitly, mathematically existent entity. Saying that $\mathrm{V}$ is the union of $V_{0}, V_{1}, V_{2}, \ldots$ is like saying that wholeness is, or contains, all the parts. The existence of each stage, $V_{x}$ for any ordinal $x$ in $V$, follows from the ZFC set of axioms. It is interesting to note, however, that the existence of $V$ itself cannot be shown from the ZFC set of axioms, since $V$ is not a set. In other words, $V$ in its totality cannot be shown to be an explicitly existing mathematical object. 
The fact that all its stages can be shown to exist seems to imply that $V$ should exist as well, however, its existence cannot be explicitly shown.

It should be stressed that our description of $V$ is but a description of a feature of wholeness and far from a complete description of wholeness. Such a description cannot be expressed in explicit terms because the nature of wholeness is not reducible to the explicit. Our description in terms of $V$ only refers to the explicit parts and states that it contains all parts but is more than that. This way of referring to wholeness is employed because the aim of this theory is to account for the existence and dynamics of all explicitly existent parts of reality.

\section{B. The wholeness preserving movement, $j$}

As argued earlier, one of the fundamental concepts upon which we wish to base a theory from wholeness is the wholeness preserving movement. This means that we wish to postulate the existence of such a movement in order to enable a prediction of quantifiable characters of an experimentally verifiable entity in terms of the wholeness preserving movement. It turns out that the Wholeness Axiom can be seen as expressing the wholeness preserving movement. The Wholeness Axiom [19] is the assumption that there is a nontrivial elementary embedding, $j$, from the mathematical universe, $V$, to itself:

$$
j: V \rightarrow V
$$

For $j$ to be an elementary embedding means that if some formula, $\phi(X)$ holds true in the domain, which for $j$ is $V$, then $\phi(j(X))$ must hold true in the codomain, which is also $V$ in this case. In other words, $j$ is reflective and truth preserving. It can be said to be wholeness preserving because due to its non-triviality it actually changes parts while preserving all structures in $V$.

Definition (9) can be axiomatized in a consistent manner $^{6}$ so that $\mathrm{ZFC}+\mathrm{WA}$ is obtained. This is done by adding $j$ to the language $\{\in\}$, so that we have $\{\in, j\}$ and saying that the ZFC set of axioms is valid with the addition that all instances of Separation and no instance of Replacement ${ }^{7}$ are valid for $j$, and the addition of axioms that express $j$ as a nontrivial elementary embedding from $V$ to itself (Nontriviality axiom and Elementarity axioms). The meaning of this in terms of wholeness will be discussed in the succeeding sections.

The Wholeness Axiom was developed to prove the existence of all large cardinals, in mathematics. And so, elementary embeddings, such as $j$, are closely connected with infinities. One of the aspects they have in common with infinities is that they disclose precisely the

\footnotetext{
${ }^{6}$ despite Kunen's theorem 23]

7 The Separation and Replacement schema are axioms contained in the ZFC set of axioms.
}

self-similar, or holographic, type of order we attribute to wholeness and find in the quantum theory. An example of self-similarity for the real numbers is that there are as many real numbers in, say, the interval between 0 and 1, as there are on the whole line. Elementary embeddings can be seen as displaying perhaps the ultimate form of self-similarity because one cannot distinguish a part from the whole with any definable formula.

\section{The critical point $\kappa$}

The introduction of the wholeness preserving movement, mathematically formulated as the Wholeness Axiom, brings about some interesting consequences. Of primary importance is the arising of a critical point. This happens because the axioms for $j$ ensure that there is a least ordinal moved by $\mathrm{j}$. This ordinal is denoted $\kappa$ and is called the critical point of $j$. In particular, we see that Nontriviality for $j$ asserts that there has to be some set $X$ for which $j(X) \neq X$, and so, there has to be some smallest ordinal for which this condition holds true. The restriction of $j$ to any set of a rank less than $\kappa$ is the identity. In other words, for any set $Y$, of rank less than $\kappa, j(Y)=Y$. Being an elementary embedding, $j$ is truth preserving. This means that properties, operations, and relations that hold true for some sets $X_{1}, X_{2}, \ldots \in V$ also hold true for $j\left(X_{1}\right), j\left(X_{2}\right), \ldots \in V$. This property of the elementary embedding makes it possible for us to say more about $\kappa$. It turns out, for instance, that $j(\kappa)>\kappa$ (see the Appendix) and that $\kappa$ is an infinite cardinal with all the large cardinal properties [19]. In can be mentioned that, as described in Section 4.E, thanks to $\kappa, j$ contains a lot of creative or generative power. This might become useful for physical theories.

\section{Separation and no Replacement for $j$}

Recalling and recapitulating some features of wholeness we can say that wholeness is indescribable, it is more than the sum of parts, and yet it is locally accessible and that through which every part emerges. All of these features are represented in $\mathrm{ZFC}+\mathrm{WA}$. In order to see this, it is of interest to note that $\mathrm{ZFC}+\mathrm{WA}$ does not restrict wholeness, or the wholeness preserving movement, to some explicitly existent mathematical object. All it does along those lines is to assume the existence of a wholeness preserving movement. The wholeness preserving movement is itself never defined by some specific formula. In fact, it cannot be defined within set theory. $j$ is neither a set nor a proper class. The fact that $j$ is not a set follows from $j$ being defined on all of $V$. To see why it is not a proper class either we need to take a look at the axioms of Separation and Replacement.

When axiomatizing the wholeness preserving movement, $j$, it is said that all instances of Separation and no instance of Replacement are valid for $j$. Given a set, 
$A \in V$, Separation allows us to talk of a subset of $A$ in which some property is true for all the elements of that subset. So, $j$ having all instances of Separation means that for all properties, $P$, depending on $j$, we can take any set, $A$, and look at the subset of $A$ containing all elements for which $P$ is true. That subset of $A$ is itself a set. Separation for $j$ makes $j$ interesting, powerful, and promising for a further development in terms of a physical theory, because it allows $j$ to act locally, meaning on any particular set. This allows us to use $j$ in local descriptions. What $j$ does on the entire mathematical universe, it also does locally on any set.

Replacement, on the other hand tells us that for any set $A$ and any rule that associates with each element $x$ of $A$ a set $Y_{x}$ there is a set $B$ that consists precisely of all $Y_{x}$, where $x \in A$. Replacement is useful for class functions. Class functions are functions that are not sets but proper classes defined on some proper class, such as for instance on $V$. Replacement guarantees that the range of a class function being restricted to some set is also a set. The range being a set makes its rank limited. So, we can say that Replacement makes sure that there can be no definable way of going through the top of $V$ in a certain amount of steps (indicated by the rank of the domain). No instances of Replacement for $j$ tells us that for all functions, $F$, depending on $j$, there may be no set consisting of all the $Y_{x}^{\prime} s$ associated with the elements $x$ of a given set. Indeed, Separation for $j$ can be used ${ }^{8}$ to show that if $F$ is defined by letting $F(0)=\kappa, F(1)=j(\kappa)$, $F(2)=j(j(\kappa))$, etc., and letting $F(x)=\emptyset$ for any $x$ that is not a natural number, then the restriction of $F$ to the set of natural numbers has a range that goes all the way through $V$. More simply, the sequence $\kappa<j(\kappa)<$ $j(j(\kappa))<\ldots$ extends above every rank in the universe. One says that the 'critical sequence for $j$ is cofinal in the mathematical universe'. Hence $F^{\prime} s$ depending on $j$ do not exist as sets or proper classes in $V$ and may be capable of going through the top of $V$. No Replacement for $j$ assures us that $j$ is not a proper class because it is not definable by a formula within set theory. If it were definable in set theory, then by ordinary Replacement in set theory, the $F$ defined above would have the property that the range of $F$ restricted to the natural numbers would be a set. As observed above, this is not the case.

Going back to our previously mentioned features of wholeness, $j$ is indescribable and more than "the sum of the parts". At the same time it acts locally and is present in every part of $\mathrm{V}$.

\footnotetext{
8 The proof makes essential use of Kunen's theorem, see Proposition 3.6 in [19], and [23]
}

\section{E. Laver sequences-emergence of all sets in $\mathrm{V}$}

Taking the wholeness preserving movement to be fundamental it is desirable to have a way of seeing how the movement gives rise to every part. Is there a way of describing the emergence of all sets in the mathematical universe through the wholeness preserving movement? Although, strictly speaking, mathematics is in general not viewed as emerging from anything, it is possible to see all the sets in the mathematical universe as an expression of the dynamics of wholeness. In order to see how this can be done we can use Laver sequences [24]. The definition of a Laver sequence given here is generalized by, and due to, Corazza. A Laver sequence is a set $S$ of length $\kappa, \kappa$ being the critical point of $j$, that has the property that for any set $X$ in $V$ there is an elementary embedding $i: V_{\alpha} \rightarrow V_{\beta}$ such that the $\kappa$ :th term of $i(S)$ is $X$. In other words, every set in the mathematical universe can be located as the $\kappa$ :th term of an image of the Laver sequence $S$ by some elementary embedding $i$. Moreover, all such $i$ 's are derived directly from $j$ itself. Therefore, every set exists as if in seed form in the single point $S$; its existence as a set becomes apparent when the appropriately derived embedding $i$ is applied to $S$. So, given $\kappa$ we can construct a sequence such that every set in the mathematical universe can be seen to emerge from it through movement. The existence of such sequences can be shown assuming the Wholeness Axiom. Another way of looking at Laver sequences is to see them as functions $f: \kappa \rightarrow V_{\kappa}$ such that for any set $X \in V$ there is an elementary embedding, $i: V_{\alpha} \rightarrow V_{\beta}$, such that $i(f)(\kappa)=X$. As functions, Laver sequences live in the mathematical universe and may be said to be made out of parts. However, a detailed understanding of the parts does not facilitate the understanding of them. It is not until one "shines" an elementary embedding on them that they become "active" and one realizes their power to give rise to all sets. These global properties, not perceptible from the details but visible through movement, are what make them interesting. These properties may come to play a key role in finding new formative causal structures [26] for physical theories from wholeness.

In short, the Wholeness Axiom provides wholeness preserving movement. As a consequence there is a $\kappa$ with which we can form a Laver sequence from which all sets in $V$ can be seen to emerge through the movement.

\section{FROM THE WHOLENESS AXIOM TOWARDS PHYSICS}

We shall now sketch out a possible way of elaborating on and developing the approach of starting from wholeness and its here introduced mathematical formalism. However, before going into the further development of the mathematics, we note that this approach already provides us with a conceptual structure that makes it possible to evolve our intuitive understanding of the theories 
we already have, such as the quantum theory. Let us take a look at such an example.

\section{A. Insights into Heisenberg's principle of indeterminacy}

Here we argue that if an undivided wholeness is assumed, then the holistic view can provide insights into features of the quantum theory along with intuitive understanding. In order to better understand what a holistic view entails and adds, we shall, at this point, examine some fundamental differences in the concepts and requirements for reductionism and holism. A good description of these differences may be found in [27].

Reductionism presupposes the possibility of decomposition into identifiable parts. This means that we view the system that we study as essentially being made up of separate, identifiable parts, that interact with each other as described by laws. The description of the whole system is reducible to a description of its parts. In order to be able to have identifiable parts we need to have both strict causality and strict locality (or space-time continuity). And having both means that we can in principle always find identifiable parts. In other words, identifiable parts are equivalent to strict locality together with strict causality. Should either of these fail, then we will no longer be able to identify parts.

Let us now examine the holistic view. This involves using indivisible wholeness as most basic and fundamental. The holistic view assumes that there are no separate parts. Although there may be distinguishable modes on some level, on a deeper level we will see that separation and precise distinction are not possible. In other words, on that level we would not be able to find identifiable parts. As a consequence we could not have both strict causality and strict locality. We could have one of them, but that would imply that we would not be able to have the other. As we saw in the second paragraph, having both implies identifiable parts. Another alternative is that we could have neither strict causality nor strict locality. It is interesting to note that this is exactly what happens in the quantum theory [28], and can there be seen as an expression of Heisenberg's principle of indeterminacy. One can intuitively think of position and time as expressing the local aspects, while energy and momentum can be considered to express the causal aspects 27]. Assuming wholeness, the appearance of a principle such as Heisenberg's principle of indeterminacy can hereby be seen as a necessary consequence of the undivided wholeness itself. We can see why strict causality and strict locality are not simultaneously possible.

\section{B. Connection of the mathematical framework with physics}

Let us now continue by outlining, step by step, a possible way of further developing the mathematics so as to establish a correspondence with physical phenomena. A detailed exposition of the following presentation will be published elsewhere by the author.

Since physics is about describing how different processes relate to each other we are interested in seeing the kinds of processes that the wholeness preserving movement can generate and what relational structure this gives rise to. A way of generating different processes is to have $j$ interact with itself. To describe this mathematically we define the binary operation of application, $\cdot$, 29] (page 54), and apply $j$ to itself. In doing so repeatedly, we end up with a universal algebra $A_{j}$ comprised of elements such that each element is obtained by finitely many applications of $j$. Thus $j, j \cdot j, j \cdot(j \cdot j),(j \cdot j) \cdot j$ are some of the elements found in $A_{j} . A_{j}$ together with application is a free left-distributive algebra with a linear ordering on it 30]. Left-distributivity means that if $a, b$, and $c$ are elements of $A_{j}$ then $a \cdot(b \cdot c)=(a \cdot b) \cdot(a \cdot c)$. These kinds of algebras on elementary embeddings have originally been studied by Laver [30] and an introduction to them can be found in 31].

With $A_{j}$ we now have a process algebra where the processes can act on each other through application and every process is obtained from the wholeness preserving movement. We note that conceptually, this algebra fits exceptionally well into the process algebra approach advocated by Bohm and Hiley [2], 4]. Their goal is to describe nature in terms of process that originates in the dynamical holomovement of the implicate order [4]. The algebra $A_{j}$ on the elementary embeddings is precisely that. It is a process algebra, where all the elements are elements of movement generated by the wholeness preserving movement.

The next step is to note that the process algebra $A_{j}$ is, through its left-distributive structure, directly tied to braids. Left-distributivity can be said to be behind the geometry of braids 31. It turns out that the process algebra $A_{j}$ is isomorphic to special braids [31] (p 103). Special braids are obtained by letting the unit braid, 1, act on itself through braid exponentiation [31](p 28). Every braid in the braid group can be decomposed into shifted products of special braids. By looking at $A_{j}$ as the algebra of special braids we focus our attention on the structural aspect, that is, how processes are related to processes (as is usually done in physics), while steering away from the insight of what these processes really are, elementary embeddings representing the wholeness preserving movement whose innermost nature is wholeness.

In physics braids do not only turn up in certain applications like the experimentally verified statistics of anyons in the Quantum Hall effect 32 or more theoretically as Yang-Baxter operators providing useful solutions for low dimensional quantum field theory 33] or lattice 
statistical physics [34], but also in the very fundamental structure of physics itself as in, for instance, the form of braided commutativity 35. In fact, some mathematical physicists are studying braids and Hopf algebras are doing so in the hope of unifying the quantum theory with general relativity [35].

Just to give a bit more insight into how braids can be related to physics in the aforementioned approaches we follow with a few comments. One particular connection between braids and statistical mechanics [36] is usually described in terms of knots, or more generally links. However, a link can always be written in terms of braids, as the closure of a braid. Links that may be transformed into each other without the tearing of any strings, that is using the so called Reidemeister moves [37], are said to be equivalent. It turns out that a link can be seen as a lattice on which we might, for instance, place spins either at the lattice sites (spin models) or at the edges (vertex models). The procedure is then to construct models such that all lattices corresponding to equivalent links give us the same partition function. This way the partition function is a link invariant.

However, as already mentioned the connection is not limited to statistical mechanics. Depending on features such as global properties and discreteness, the topology of braids can also be connected with quantum features. In fact, the connection between statistical mechanics and knot theory was first discovered by Jones 38] by noting the similarities between von Neumann algebras and the braid relations. A von Neumann algebra is a $C^{*}$-algebra, used in algebraic approaches to the quantum theory such as [39]. Jones derived a link invariant, called the Jones polynomial, which through the connection with von Neumann algebras can be seen as providing expectation values.

The connection between knots and physics has been further explored by Kauffman [40]. And here we wish to especially point to a certain bracket polynomial 40] (p 97 ), which can be defined for links or braids. The approach taken by Kauffman in general is to start with a braid (or link) and with the help of his bracket arrive at an algebraic structure, a link invariant, which can take on physical interpretations such as expectation values. What is proposed here is an approach more along the lines of Dehorony [31] where we not only look at the algebraic structure associated with a particular braid, but the structure associated with all possible braids, and in fact from where they can be seen to originate (a structure like the process algebra $A_{j}$ ). Applying structures such as the bracket to $A_{j}$ would then provide a physical interpretation. Hence the idea is that in so doing, this approach would be able to provide a background for the many already discovered connections between braids (links and knots) and physics, as well as possibly lead to new discoveries.

The outlined proposition is schematically depicted in figure 1.

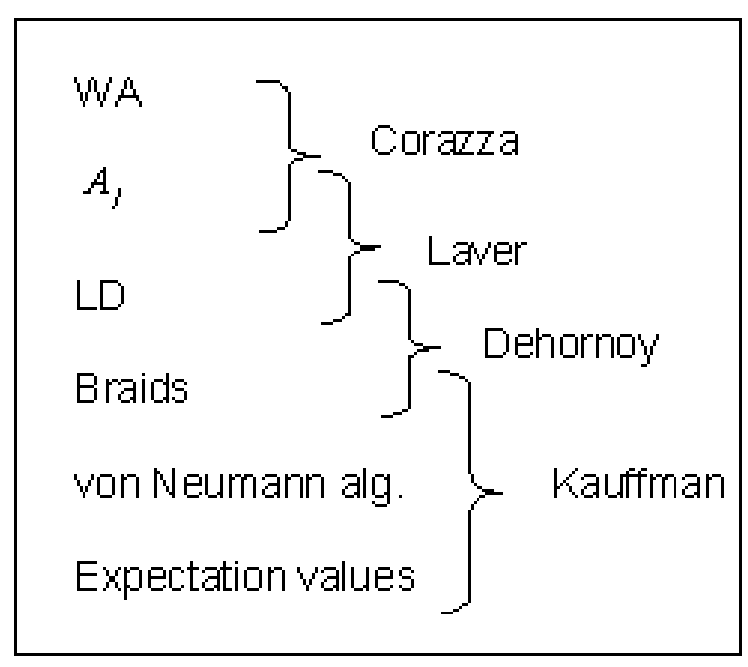

FIG. 1: Schematic picture summarizing a possible development of the approach from Wholeness. The Wholeness Axiom (WA) gives us $j$, which generates the universal algebra $A_{j}$, which is left-distributive (LD) and behind the geometry of braids, which through Kauffman's bracket can be connected to statistical mechanics and the quantum theory. The names on the right refer to mathematicians who in their work (see References) in detail describe the alluded connections.

\section{CONCLUSIONS}

In conclusion, it is suggested that a dynamical wholeness may be capable of providing a conceptual foundation for the development of physical theories. By starting out from a broader perspective, primarily motivated by the quantum theory, it provides the guidelines for the creation of a theory that may be valid where other currently existing theories reach their limits. As a fundamental concept upon which to build a theory from wholeness we suggest a wholeness preserving movement. Such a movement may be mathematically expressed in a fundamental way if the Wholeness Axiom is added to the currently established axioms of Set Theory. Such a mathematical foundation naturally allows for a description of the emergence of all parts of the mathematical universe. It also contains holistic features as indescribability, more than the sum of parts, local accessibility and the holographic type of order that we find in the quantum theory.

We have seen an example of how starting from wholeness facilitates the understanding of seemingly paradoxical features of existing theories. We have also indicated how the approach may be further developed mathematically, through a left-distributive algebra and braids, so as to connect up with physics. 


\section{APPENDIX: Proof that $j(\kappa)>\kappa$}

Let $j: V \rightarrow V$ be a non-trivial elementary embedding from the mathematical universe to itself, with $\kappa$ as its critical point. Since $j$ is by definition non-trivial $j(\kappa) \neq$ $\kappa$. Let us assume $j(\kappa)<\kappa$, and say that $j(\kappa)=y$. Then since $\kappa$ is the smallest ordinal moved by $j$ and $y<\kappa$ it must mean that $j(y)=y$. Because of elementarity ordinal order is preserved, meaning that if $y<\kappa$ then $j(y)<j(\kappa)$. But from the above we see that $j(y)$ cannot be lesser than $j(\kappa)$ because $j(y)=y=j(\kappa)$. And so we conclude that $j(\kappa)<\kappa$, is not consistent. Therefore it must be so that $j(\kappa)>\kappa$.

\section{Acknowledgements}

I would like to thank Paul Corazza for his guidance as well as B.J. Hiley, E. Sjöqvist, D. Abbott, C-G. Granqvist, P. Nadel-Turoński, P. Hammerstein, E. Palmgren, J. L. Garcia-Palacios, E.D. Avendaño Soto, S. Mirbt, and D. Hérisson for discussions or comments.
[1] N. Bohr, Atomic Theory and the Description of Nature (Cambridge University Press, Cambridge, p 11, 1934).

[2] D. Bohm and B.J. Hiley, The Undivided Universe An Ontological Interpretation of Quantum Theory, (Routledge, London and New York, 1993).

[3] A. Aspect, P. Grangier, and G. Roger, Phys. Rev. Lett. 49, 91-94, (1982).

[4] D. Bohm, Wholeness and the Implicate Order (Routledge, London and New York 1980).

[5] M. Sachs, From atomism to Holism in 21st century physics,Annales de la Foundation de Luis de Broglie, 26, no special, 389-397, (2001).

[6] D. Bohm, A Suggested Interpretation of the Quantum Theory in Terms of "Hidden Variables, I and II, Phys. Rev. vol 85 (1952), p 166-193.

[7] P. R. Holland, The Quantum Theory of Motion-An account of the de Broglie-Bohm causal interpretation of quantum mechanics, Cambridge University Press (1993).

[8] N. Bohr, Atomic Physics and Human Knowledge, Science Editions, New York (1965).

[9] D. Bohm, Hidden variables and the implicate order, published in Quantum Implications: Essays in Honur of David Bohm ed. by B.J. Hiley, F.D. Peat, Routledge and Kegan Paul Ltd, London (1987).

[10] D. Bohm, Meaning as being in the implicate order philosophy of David Bohm: a conversation, published in Quantum Implications: Essays in Honur of David Bohm ed. by B.J. Hiley, F.D. Peat, Routledge and Kegan Paul Ltd, London , (1987).

[11] D. Dürr, S. Goldstein, N. Zanghi, Quantum Mechanics, Randomness, and Deterministic Reality. Physics Letters A 172 (1992) 6-12.

[12] D. Dürr, S. Goldstein, N. Zanghi, Quantum Equilibrium and the Origin of Absolute Uncertainty. Journal of Statistical Physics, Vol 67 (1992), p. 843-907.

[13] B. J. Hiley, From the Heisenberg Picture to Bohm: a New Perspective on Active Information and its relation to Shanon Information, published in Proc. Conf. Quantum Theory: reconsiderations of foundations, ed. A. Khrennikov, p. 141-162, Växjö University Press, Sweden (2002).

[14] C. E. Shannon, A Mathematical Theory of Communication, The Bell System Technical Journal, vol 27, p 379423, 623-656, July, October, (1948).

[15] Primas Hans, Chemistry, Quantum Mechanics and Reductionism-Perpectives in Theoretical Chemistry (Springer-Verlag, Berlin Heidelberg New York, chapter
6, 308-355, 1981).

[16] P. W. Anderson, Science 177, no 4047, (1972).

[17] G. W. F. Hegel, Hegel's Logic : Being part one of the Encyclopedia of the philosophical sciences (1830) / with foreword by J. N. Findlay ; transl. by William Wallace (Oxford University Press, Oxford 1975).

[18] D. Bohm and F. D. Peat, Science, order, and creativity (Routledge, London and New York 2000).

[19] P. Corazza, Ann. Pure App. Logic, October, 157-260, (2000).

[20] E. Zermelo, I. Math. Annalen, 65, 261-281, (1908), (English translation by Stefan Bauer-Mengelberg in J. van Heijenoort, From Frege to Gödel (Cambridge Harvard University Press 1967))

[21] A. Fraenkel, Math. Annalen, 86, 230-237, (1922).

[22] http://mathworld.wolfram.com/ZermeloFraenkelAxioms.html

[23] K. Kunen, J. Symbolic Logic, 36, no 3, 407-413, (1971).

[24] R. Laver, Israel J. Math, 29, no 4, 385-388, (1978).

[25] A. Lerda, Anyons: Quantum Mechanics of Particles with Fractional Statistics (Springer-Verlag, Berlin-HeidelbergNew York, 1992).

[26] A. Baracca, D. J. Bohm, B. J. Hiley, A. E. G. Stuart On Some New Notions Concerning Locality and Nonlocality in the Quantum Theory Il Nuovo Cimento, Vol 28 B, N. 2, August 11th 1975, p 453-466.

[27] D. Bohm, Quantum Theory (Second Edition, PrenticeHall Inc, chapter 8, 144-172, 1952).

[28] J. S. Bell, Speakable and Unspeakable in Quantum Mechanics (Cambridge University Press, Cambridge chapter $2,1987)$.

[29] P. Corazza, Submitted to Annals Of Pure And Applied Logic (July 15, 2002).

[30] R. Laver, Adv. Math 91, 209-231, (1992).

[31] P. Dehornoy, Braids and Self-Distributivity (Birkhäuser Verlag, Basel-Boston-Berlin, 2000).

[32] R. E. Prague and S. M. Girvin, The Quantum Hall Effect (Springer-Verlag, Berlin, 1990).

[33] C. N. Yang, Phys. Rev. Lett. 19, 1312 (1967).

[34] R. J. Baxter, 70, no 1, 193-228 (1972).

[35] S. Majid, Foundations of quantum group theory (Cambridge Univ. Press, Cambridge 1995).

[36] F. Y. Wu, Knot theory and statistical mechanics, Reviews of Modern Physics, 64, No. 4, October 1992, 1099-1131.

[37] K. Reidemeister, Knotentheorie, Chelsea, New York, 1948; English translation, ed. by L.F. Boron, C.D. Christenson and B.A. Smith, Knot Theory, BCS Associates, 
Moscow, Idaho, 1983.

[38] V.F.R. Jones, A polynomial invariant for links via von Neumann algebras, Bull.Am.Math.Soc. 12, 103-112.

[39] Rudolf Haag, Local Quantum Physics-Fields, Prticles, Algebras, 2nd revised edition, Springer-Verlag, Berlin-
Heidelberg-New York, 1996.

[40] L. H. Kauffman, Knots and Physics (World Scientific, Singapore, 1991). 